\title{
Assessing the time constancy of the proton-to-electron mass ratio by precision ro-vibrational spectroscopy of a cold molecular beam
}

\author{
L. Santamaria, ${ }^{1}$ V. Di Sarno, ${ }^{1}$ I. Ricciardi, ${ }^{1}$ S. Mosca, ${ }^{1}$ M. De Rosa,${ }^{1}$ G. \\ Santambrogio, ${ }^{2,3}$ P. Maddaloni, ${ }^{1,4, \star}$ and P. De Natale ${ }^{4,5}$ \\ ${ }^{1}$ CNR-INO, Istituto Nazionale di Ottica, Via Campi Flegrei 34, 80078 Pozzuoli, Italy \\ ${ }^{2}$ CNR-INO, Istituto Nazionale di Ottica, Via N. Carrara 1, 50019 Sesto Fiorentino, Italy \\ ${ }^{3}$ Fritz-Haber-Institut der Max-Planck-Gesellschaft, Faradayweg 4-6, 14195 Berlin, \\ Germany \\ ${ }^{4}$ INFN, Istituto Nazionale di Fisica Nucleare - Sez. di Firenze, Via G. Sansone 1, 50019 \\ Sesto Fiorentino, Italy \\ ${ }^{5}$ CNR-INO, Istituto Nazionale di Ottica, Largo E. Fermi 6, 50125 Firenze, Italy \\ ${ }^{\star}$ Corresponding author
}

\begin{abstract}
We report the design of an experiment that aims to constrain, over a-fewyear timescale, the fractional temporal variation of the proton-to-electron mass ratio, $\beta=m_{p} / m_{e}$, at a level of $10^{-15} / \mathrm{yr}$ by means of a spectroscopic frequency measurement on a beam of cold $\mathrm{CF}_{3} \mathrm{H}$ molecules. This is extracted from a buffer-gas-cooling source and then collimated by means of an electrostatic hexapole lens. Employed in a two-photon Ramsey-fringes interrogation scheme, the probe source is based on a mid-infrared quantum cascade laser, phase-locked to a specially-developed optical frequency comb that is ultimately referenced to the Cs primary standard via an optical fiber link.
\end{abstract}

Keywords: proton-to-electron mass ratio, buffer gas cooling, two-photon Ramsey spectroscopy 


\section{Introduction}

Thanks to the breakthrough technologies originating respectively from the world of ultracold quantum gases and that of femtosecond laser frequency combs during the last decades, methods of precision spectroscopy have advanced to the point where atomic/molecular transition frequencies can be determined with an astonishing precision (down to a few parts in $10^{-18}$ ), to such an extent that detecting the influence of fundamental new physics at the eV energy scale is now within reach [1]. In this frame, more and more challenging experiments are underway, aiming at testing nature symmetries and constants with unprecedented sensitivity. In particular, in the last few years a strong interest has focused on the possibility that what we know as the fundamental physical constants might show variations over cosmological time scales. Such an effect arises quite naturally in modern theories (Lie Groups, String/M Theories, ... ) attempting either to establish a Grand Unification Theory beyond the Standard Model or to reconcile this latter and General Relativity in a Theory of Everything [2, 3, 4, 5]. Since variation of dimensional constants cannot be distinguished from that of the units, it makes more sense to consider changes of dimensionless parameters. The prime target is the fine structure constant, $\alpha=e^{2} /\left(4 \pi \varepsilon_{0} \hbar c\right)$, which defines the scale of quantum electrodynamics; the second prominent quantity is the proton-to-electron mass ratio, $\beta=m_{p} / m_{e}$, which characterizes the strength of strong interaction in terms of the electro-weak one. While in the former case the temporal stability is conveniently probed through atomic transitions [6], the $\beta$ ratio is more accurately addressed with molecular systems where resorting to the inaccurate nuclear Schmidt model is bypassed [7, 8]. One approach is to compare the wavelengths of molecular lines measured in the present epoch on Earth with the corresponding ones from astronomical objects at high redshifts $[9,10,11]$. The most up-to-date constraint for $\Delta \beta / \beta$ is $(0.0 \pm 1.0) \cdot 10^{-7}$, deduced from the observation of radio-frequency transitions of methanol in the PKS1830-211 galaxy at redshift $z=0.89$ (corresponding to a look-back time of 7 billion years). Assuming a linear temporal variation, this implies a fractional variation $\dot{\beta} / \beta$ on the order of $10^{-17} \cdot \mathrm{yr}^{-1}$, consistent with a null result [12]. A second approach relies on precision molecular spectroscopy experiments entirely carried out in the laboratory, with the inherent advantage that all systematic effects can be meticulously controlled. Different schemes have been proposed in this frame, including an ammonia fountain [13], molecular hydrogen ions confined in radio-frequency traps [14], 
and magnetically trapped cold XH (X: alkali-earth atom) molecules [15].

\section{The overall proposal}

Following the scheme originally implemented by A. Shelkovnikov and coworkers with a conventional supersonic beam of $\mathrm{SF}_{6}$ [16], our proposal is to measure the frequency $\nu_{\text {vib }}$ of a given molecular ro-vibrational transition relative to the clock hyperfine transition $\nu_{\text {hyp }}$ in the Cs electronic ground state $\left(\left|F=4, m_{F}=0\right\rangle \leftrightarrow\left|F=3, m_{F}=0\right\rangle\right)$. Such frequencies scale respectively as $[3]$

$$
\begin{gathered}
\nu_{\mathrm{vib}}(\mathrm{S})=K_{\mathrm{S}} \cdot \beta^{-1 / 2} \cdot R y \\
\nu_{\mathrm{hyp}}(\mathrm{Cs})=K_{\mathrm{Cs}} \cdot\left(\frac{\mu_{\mathrm{Cs}}}{\mu_{B}}\right) \cdot \alpha^{2} \cdot F(\alpha) \cdot R y
\end{gathered}
$$

where $\mathrm{S}$ indicates a generic molecular species. Here, $K_{\mathrm{S}}$ and $K_{\mathrm{Cs}}$ are proportionality constants, $\mu_{\mathrm{Cs}}$ is the magnetic dipole of the Cs nucleus, $\mu_{B}$ the Bohr magneton, Ry the Rydberg constant, and $F(\alpha)$ is a dimensionless function accounting for relativistic effects in Cs, whose dependence on $\alpha$ is to the power of 0.83 . From these formulas the following relationship between our observable $\nu_{\text {vib }} / \nu_{\text {hyp }}$ and $\beta$ is derived

$$
\frac{1}{\frac{\nu_{v i b}(\mathrm{~S})}{\nu_{h y p}(\mathrm{Cs})}} \frac{\partial\left[\frac{\nu_{v i b}(\mathrm{~S})}{\nu_{h y p}(\mathrm{Cs})}\right]}{\partial t}=-\frac{1}{2 \beta} \frac{\partial \beta}{\partial t}-\frac{2.83}{\alpha} \frac{\partial \alpha}{\partial t}-\frac{1}{\frac{\mu_{\mathrm{Cs}}}{\mu_{B}}} \frac{\partial\left[\frac{\mu_{\mathrm{Cs}}}{\mu_{B}}\right]}{\partial t}
$$

which, in combination with the current constraints of $\dot{\alpha} / \alpha=(-1.6 \pm 2.3)$. $10^{-17} \mathrm{yr}^{-1}$ and $d / d t\left[\ln \left(\mu_{\mathrm{Cs}} / \mu_{B}\right)\right]=(-1.9 \pm 4.0) \cdot 10^{-16} \mathrm{yr}^{-1}$, as inferred from a 6-year record of increasingly precise atomic clock frequency comparisons [17], can be used to set an upper limit to the temporal variation of $\beta$.

To enhance the spectroscopic interrogation time, which sets the ultimate resolution achievable in a single measurement, a Ramsey-fringes technique will be adopted [18]. In this scheme one first wants to minimize the fringe periodicity $P$, given by the ratio of the mean longitudinal speed of the molecules in the beam, $u$, to the distance $D$ between the two interaction zones: $P=u /(2 D)$. Second, a high fringe contrast is desirable, which can be accomplished by reducing the velocity dispersion, $(\Delta u)^{-1} \sim 1 / \sqrt{T_{\text {trans }}}$, with $T_{\text {trans }}$ being the translational temperature of the molecular beam. As 
discussed later, a low rotational temperature, $T_{\text {rot }}$, is also desirable; indeed, this increases the population in states with low rotational quantum numbers, which can be exploited to enhance the strength of the final spectroscopic absorption feature. While for a generic molecular beam the inequality chain $T_{v i b} \geq T_{\text {rot }} \geq T_{\text {trans }}$ holds, under typical operating conditions of our experiment (see Section 3.2), these three temperatures roughly coincide with each other so that we will simply speak of the beam temperature $T$. In conclusion, a great enhancement in the ultimate accuracy can be achieved if a beam of slow and cold molecules is used. In the optical domain, the Ramsey-fringes method has to be associated with a sub-Doppler technique in order to avoid a scrambling of the fringe pattern. Opting for two-photon spectroscopy, the most efficient configuration is represented by the two-zone geometry where the molecular beam interacts successively with two standing waves generated from the probe laser inside a single folded Fabry-Perot enhancement cavity comprising four mirrors in a $\mathrm{U}$ configuration [19, 20].

In the following, we shall describe the main building blocks of the experiment starting from those which, already constructed, are about to be characterized (Section 3) and ending with those that are being developed (Section 4).

\section{Design of the molecular beam}

The aim is to generate a molecular beam with intense flux, low temperature, and reduced longitudinal speed. This will be attained by combining buffer-gas cooling (BGC) and Stark manipulation. Since BGC operates with nearly all species, potentially any molecule with a relatively high electric dipole moment (EDM) and a favorable two-photon transition may be chosen for the experiment.

\subsection{The molecular choice}

The fluoroform $\left(\mathrm{CF}_{3} \mathrm{H}\right)$ molecule exhibits both these features. First, a fair permanent EDM value, $\mu=1.67$ Debye, makes it suitable for manipulation with external electric fields. Second, it has a fundamental, strong rovibrational band $\left(\mathrm{CF}_{3}\right.$ degenerate stretch, $\left.v_{5}\right)$ at 8.63 micron $\left(1158.75 \mathrm{~cm}^{-1}\right)$ [21], where high-performance quantum cascade lasers (QCLs) are available and effective optical frequency combs can be developed. Shown in Fig. 1 is the two-photon transition identified for the spectroscopic measurement, $|\mathbf{0}\rangle \equiv\left|v_{5}=0, J=1, K=1\right\rangle \rightarrow|\mathbf{1}\rangle \equiv\left|v_{5}=1, J=2, K=2, l=-1\right\rangle \rightarrow$ 
$|2\rangle \equiv\left|v_{5}=2, J=2, K=1\right\rangle$, where $J$ is the total angular momentum quantum number, $K$ describes the projection of the vector $\boldsymbol{J}$ onto the molecular axis and $l$ is an additional quantum number (exclusively for rotational states pertaining to degenerate vibrational levels) from the Coriolis coupling interaction [22]. To determine the detuning $\delta$ of the virtual level from the intermediate one, we start with the general consideration that the energy of a given ro-vibrational level is the sum of a vibrational and a rotational term, $E_{\text {ro-vib }}=E_{v i b}+E_{\text {rot }}$. In addition, for an oblate symmetric-top molecule and retaining only the leading terms, the rotational energy can be expressed as $E_{r o t} \equiv E(J, K)=B J(J+1)+(C-B) K^{2}-2 \xi K l$. Then, using the vibrational anharmonicity value $E_{v i b}\left(2 v_{5}\right) / 2-E_{v i b}\left(v_{5}\right)=19.4865 \mathrm{GHz}$ [23], $B=10.425 \mathrm{GHz}$ and $C=5.716 \mathrm{GHz}[24]$, and $\xi=4.06857 \mathrm{GHz}[25]$, $\delta=\left[E_{\text {ro-vib }}(|\mathbf{2}\rangle)-E_{\text {ro-vib }}(|\mathbf{0}\rangle)\right] / 2-\left[E_{\text {ro-vib }}(|\mathbf{1}\rangle)-E_{\text {ro-vib }}(|\mathbf{0}\rangle)\right]=-3.51$ $\mathrm{GHz}$ is found. In conjunction with the expected favorable transition dipole moments [26] as well as with the cooling-induced population enhancement of low-lying rotational levels, this relatively small detuning makes the chosen two-photon transition profitable.

\subsection{Buffer gas cooling}

In this first stage, both translational and internal degrees of freedom of the desired molecular species $\mathrm{S}$, at initial temperature $T_{0}$, are cooled in a cryogenic cell via elastic collisions with a thermal bath of helium (buffer gas) at temperature $T_{H e}$ and density $n_{H e}[27,28]$. After a characteristic number of collisions $N_{\text {coll }}$, the temperature of $\mathrm{S}, T\left(N_{\text {coll }}\right)=T_{H e}+\left[T_{0}-\right.$ $\left.T_{H e}\right] \exp \left[-N_{\text {coll }} / k\right]$, becomes arbitrarily close to equilibrium with the buffer gas; here $k=\left(m+m_{H e}\right)^{2} /\left(2 m \cdot m_{H e}\right)$, where $m\left(m_{H e}\right)$ is the mass of $\mathrm{S}(\mathrm{He})$. Then, a beam of $\mathrm{S}$ is formed by letting both He and $\mathrm{S}$ particles escape into a high-vacuum environment via an orifice of radius $d$. Expansion typically occurs in the partially hydrodynamic regime determined by a Reynolds number, $R e$, between 1 and 100 (we recall that, in general, Re is defined as the ratio of inertial to viscous forces in a fluid flow). In the BGC case, one has $R e \equiv 8 \sqrt{2} f_{0, H e} \sigma_{H e}\left\langle v_{0, H e}\right\rangle^{-1} d^{-1}$, with $f_{0, H e}$ being the input flux of He, $\sigma_{H e}$ the elastic cross section for cold He collisions and $\left\langle v_{0, H e}\right\rangle=\sqrt{\left(8 k_{B} T_{H e}\right) / \pi m_{H e}}$ the average thermal velocity of the buffer gas in the cell. Physically, the partially hydrodynamic choice corresponds to the best compromise between a conventional effusive beam $(R e<1)$, exhibiting a reduced mean forward velocity $u \equiv v_{\|}$, and a supersonic one $(R e>100)$, characterized by a low temperature (i.e. spread around $v_{\|}, \Delta v_{\|}$). In this intermediate regime, both 


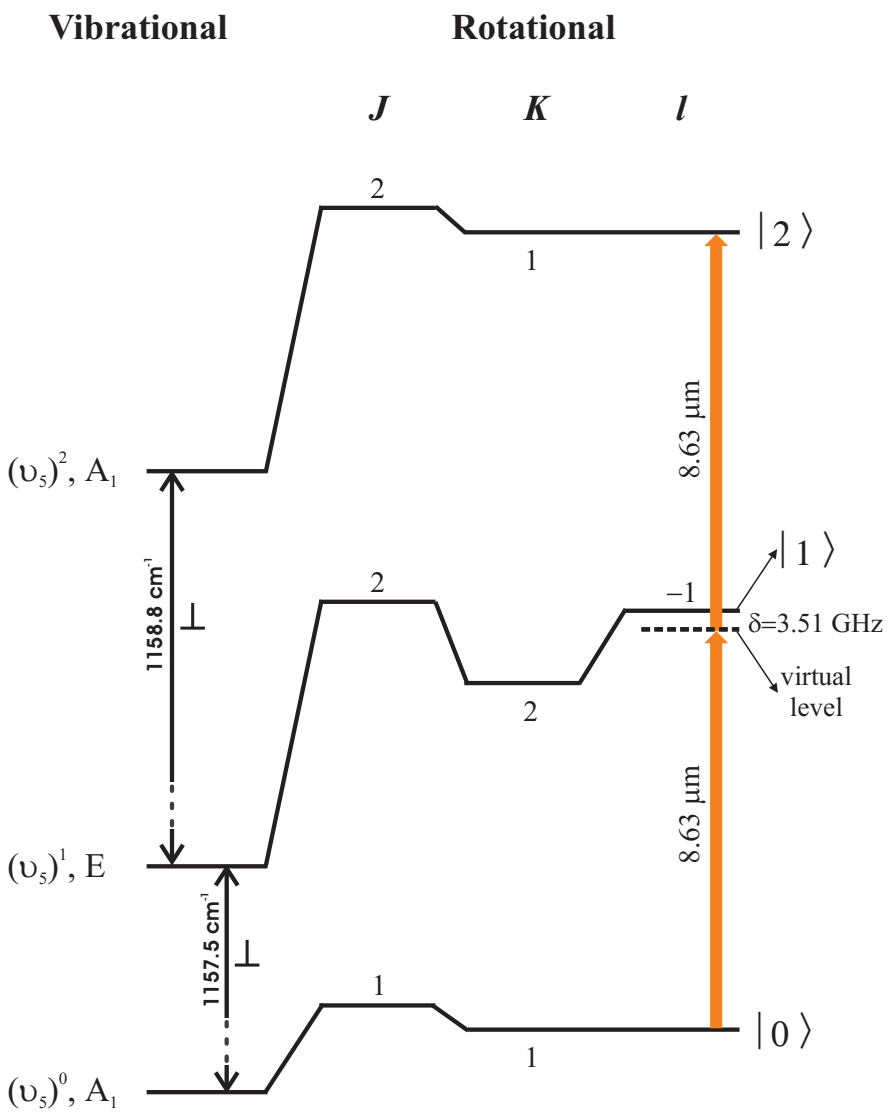

Figure 1: $\mathrm{CF}_{3} \mathrm{H}$ two-photon transition selected for the Ramsey interrogation. The symbol $\perp$ indicates that the vibrational transition moment is perpendicular to the top axis; accordingly, the following selection rules hold: $\Delta K \equiv K_{i}-K_{f}= \pm 1 ; \Delta J=0, \pm 1$. In addition, for $\left(A_{1} \rightarrow E\right)$-type transitions, the following selection rules are valid for the quantum number $l: \Delta l=+1$ for $\Delta K=-1 ; \Delta l=-1$ for $\Delta K=+1$; the reverse is true for $\left(E \rightarrow A_{1}\right)$-type transitions.

molecular kinetics (sufficient to characterize effusive beams) and fluid-like behavior (required to describe supersonic beams) determine the beam properties; for $1 \lesssim R e \lesssim 10$, the mean longitudinal speed can be expressed as a function of $\left\langle v_{0, H e}\right\rangle$ and the average thermal velocity of $\mathrm{S}$ in the cell, $\left\langle v_{0}\right\rangle=\sqrt{\left(8 k_{B} T_{H e}\right) / \pi m}:[28]$

$$
v_{\|}=1.2\left\langle v_{0}\right\rangle+0.6\left\langle v_{0, H e}\right\rangle R e \frac{m_{H e}}{m}
$$


while the transverse and longitudinal velocity spreads remain roughly constant at the effusive-regime value

$$
\Delta v_{\|} \simeq \Delta v_{\perp}=\sqrt{\frac{8 k_{B} T \ln 2}{m}} \simeq 1.5\left\langle v_{0}\right\rangle
$$

with $T \simeq T_{H e}$, whereupon the angular divergence is calculated as

$$
\Delta \theta=\frac{180^{\circ}}{\pi} \arctan \left(\frac{\Delta v_{\perp}}{2 v_{\|}}\right)
$$

The BGC apparatus is shown in Fig. 2. It is based on a two-stage pulse tube (PT) cryocooler (Cryomech PT415) housed in a stainless-steel vacuum chamber [29]. The first (second) stage of the PT refrigerator reaches a temperature of $35 \mathrm{~K}(4.2 \mathrm{~K})$ provided that the corresponding heat load is kept below $40 \mathrm{~W}(1.5 \mathrm{~W})$; for this purpose, each plate is enclosed in a round gold-plated copper radiation shield. Capillary filling, regulated upstream by two flow meters, is used to inject both $\mathrm{S}$ and the buffer gas, coming from room-temperature bottles, into the BGC cell. While the $\mathrm{S}$ pipeline (made of stainless-steel) is thermally isolated from both the PT stages, the buffer gas line comprises four connected segments: the first one is a stainless-steel pipe made of several windings (this increases the duct length, thus reducing the heat conductance of the gas line); then, a copper tube in good thermal contact with the first PT plate ensures cooling of the He gas down to $35 \mathrm{~K}$; the third segment is again made of stainless-steel in order to minimize thermal exchanges between the two PT stages; finally, a copper pipe in good thermal contact with the second PT plate guarantees cooling of the He gas down to $4.2 \mathrm{~K}$. The BGC cell, also made of gold-plated copper to maximize thermal conductivity while minimizing desorption from the walls, is in thermal contact with the $4.2-\mathrm{K}$ plate and has internal dimensions of 60 Height $(H) \times 60$ Width $(W) \times 20$ Length $(L) \mathrm{mm}^{3}$. In particular, the $L$ value was chosen so as to satisfy the criterion for an efficient thermalization, $L>N_{\text {coll }} \cdot \lambda$, where $\lambda=\pi d^{2}\left\langle v_{0, H e}\right\rangle /\left(4 \sigma f_{0, H e} \sqrt{m / m_{H e}}\right)$ represents the mean free path of the $\mathrm{S}$ molecules (here, $\sigma$ denotes the elastic cross section for cold S-He collisions). To preserve a low heat conductance against the injection of the warm molecules, the back side of the cell has a large aperture plugged by a Kapton foil, provided with an entrance hole punched against the $\mathrm{S}$ pipe. Moreover, a proportional-integral-derivative (PID) controller having a silicon diode thermometer as input sensor and an electric heater as output 
transducer (both devices being secured to the $\mathrm{S}$ pipe), is employed to avoid possible condensation of S. A similar system is used to finely tune the cryogenic cell temperature. To keep the pressure within the radiation shields as low as possible (collisions of guided molecules with background gas are practically negligible for pressures lower than $\sim 10^{-6}$ mbar), the internal surface of the inner shield is covered with a layer of activated charcoal that, at cryogenic temperatures, acts as a pump for helium and non-guided molecules (with a pumping speed of a few thousands $1 \cdot \mathrm{s}^{-1}$ ); the gas (mainly helium buffer gas) adsorbed by the charcoal is released during warm up of the cryogenic system and then pumped out of the vessel by a turbo-molecular pump. Finally, the vacuum chamber is equipped with a number of optical windows for spectroscopic interrogation.

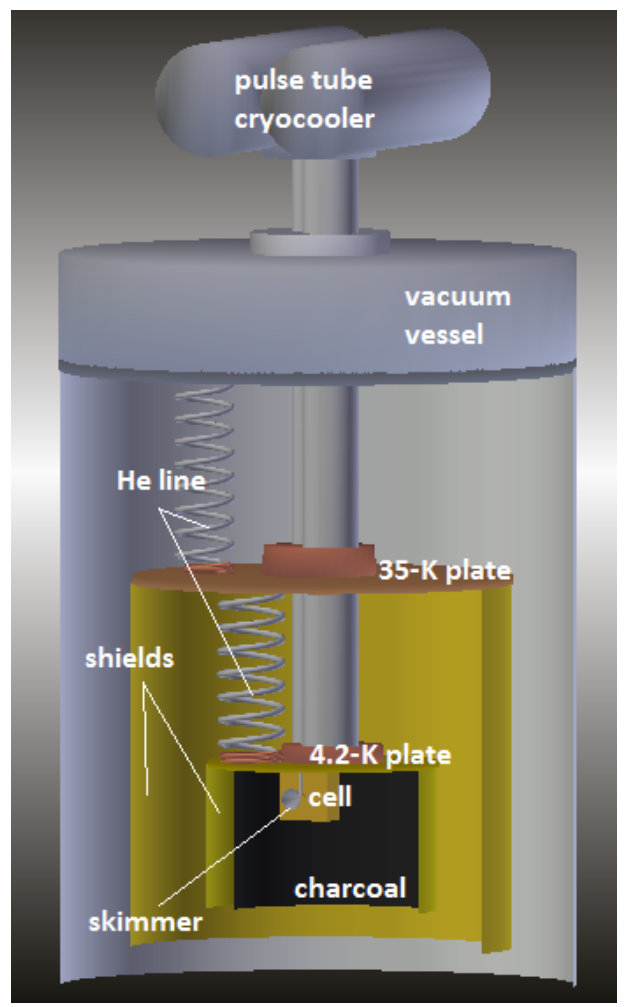

Figure 2: Buffer gas cooling apparatus.

The relevant figures expected for the fluoroform beam are summarized in Table 1. In particular its flux, $\mathcal{F}$, is estimated by regarding the extraction process as a competition between diffusion to the wall and pump-out, 
with characteristic times $\tau_{d i f f}=64 W H \sigma f_{0, H e} /\left(9 \pi^{2} d^{2}\left\langle v_{0, H e}\right\rangle^{2}\right)$ and $\tau_{\text {pump }}=$ $4 W H L /\left(\pi d^{2}\left\langle v_{0, H e}\right\rangle\right)$, respectively [28]. If $\gamma \equiv \tau_{\text {diff }} / \tau_{\text {pump }} \ll 1$, then one obtains $[30]$

$$
\mathcal{F} \simeq \frac{\pi d^{2}}{W \cdot H} f_{0}
$$

Here, $f_{0}$ denotes the input flux of $\mathrm{S}$, while the ratio between the area of the exit hole and that of the cell accounts for freezing of the $\mathrm{S}$ molecules under impact with the walls. The divergence figure, as determined by Eq. 6 , can be further narrowed down to $\Delta \theta_{\text {skim }}$ by the use of a skimmer which, however, inevitably reduces the beam flux to $\mathcal{F}_{\text {skim }}$; a good compromise is obtained using a 2-mm-input-diameter skimmer $\left(2 \mathrm{~cm}\right.$ length and $35^{\circ}$ full aperture angle) placed at $3 \mathrm{~cm}$ from the cell hole. Stopping the peripheral, less useful beam portions (i.e. the ones containing molecules with higher radial velocities) also reduces the vacuum degradation in the spectroscopic interrogation chamber.

We now evaluate the fraction of the molecular beam flux in the starting level of the selected two-photon transition. The probability $p_{e, \boldsymbol{v}, \boldsymbol{r}}$ of finding a molecule in the state $|e, \boldsymbol{v}, \boldsymbol{r}\rangle$ is given by $\mathcal{F}_{e, \boldsymbol{v}, \boldsymbol{r}}=p_{e, \boldsymbol{v}, \boldsymbol{r}} \cdot \mathcal{F}_{\text {skim }}$; here, $e, \boldsymbol{v} \equiv$ $\left(v_{1}, v_{2}, v_{3}, v_{4}, v_{5}, v_{6}\right)$ and $\boldsymbol{r} \equiv(J, K)$ represent respectively the electronic, vibrational and rotational quantum numbers. In the case of a symmetrictop molecule, and considering the electronic and vibrational ground states, $e \equiv e_{g}$ and $\boldsymbol{v}_{g} \equiv(0,0,0,0,0,0)$, one can safely assume $p_{e_{g}} \simeq p_{\boldsymbol{v}_{g}} \simeq 1$ and then write

$$
p_{e_{g}, \boldsymbol{v}_{g}, \boldsymbol{r}} \simeq p_{e_{g}} \cdot p_{\boldsymbol{v}_{g}} \cdot p_{J, K} \simeq p_{J, K}(T)=\frac{g_{J, K} e^{-E_{J, K} / k_{B} T}}{\sum_{J, K} g_{J, K} e^{-E_{J, K} / k_{B} T}}
$$

where $E_{J, K} \equiv E(J, K)$ is the rotational energy defined above and $g_{J, K}$ the corresponding degree of degeneracy. The latter is given by $g_{J, K}=(2 J+$ 1) $g_{K} g_{I}[22]$, where

$$
g_{K}= \begin{cases}1 & \text { for } K=0 \\ 2 & \text { for } K \neq 0\end{cases}
$$

and the nuclear degeneracy for molecules of point group $C_{3 v}$ is expressed as

$$
g_{I}= \begin{cases}\frac{1}{3}(2 I+1)\left(4 I^{2}+4 I+3\right) & \text { for } K \text { divisible by } 3 \text { (including zero) } \\ \frac{1}{3}(2 I+1)\left(4 I^{2}+4 I\right) & \text { for } K \text { not divisible by } 3\end{cases}
$$


Table 1: Relevant BGC parameters for a $\mathrm{CF}_{3} \mathrm{H}$ beam produced in the partially hydrodynamic regime. ${ }^{(*)} 1 \mathrm{SCCM} \simeq 4.5 \cdot 10^{17} \mathrm{molec} / \mathrm{s}$.

\begin{tabular}{ccc}
\hline$H=60 \mathrm{~mm}$ & $\sigma=10^{-18} \mathrm{~m}^{2}$ & $\gamma=0.08$ \\
$W=60 \mathrm{~mm}$ & $\sigma_{H e}=3 \cdot 10^{-19} \mathrm{~m}^{2}[31]$ & $u \equiv v_{\|} \simeq 70 \mathrm{~m} / \mathrm{s}$ \\
$L=20 \mathrm{~mm}$ & $\lambda=80 \mu \mathrm{m}$ & $\Delta v_{\|} \simeq \Delta v_{\perp} \simeq 53 \mathrm{~m} / \mathrm{s}$ \\
$d=1 \mathrm{~mm}$ & $N_{\text {coll }}=80$ & $\Delta \theta=21^{\circ}$ \\
$f_{0, H e}=1 \mathrm{SCCM}^{(\star)}$ & $\left\langle v_{0, \text { He }}\right\rangle=150 \mathrm{~m} / \mathrm{s}$ & $\mathcal{F} \simeq 4 \cdot 10^{14} \mathrm{molec} / \mathrm{s}$ \\
$f_{0}=1 \mathrm{SCCM}^{\star *}$ & $\left\langle v_{0}\right\rangle=35 \mathrm{~m} / \mathrm{s}$ & $\Delta \theta_{\text {skim }} \simeq 2^{\circ}$ \\
$T_{\text {He }}=4.2 \mathrm{~K}$ & $T \simeq 4.2 \mathrm{~K}$ & $\mathcal{F}_{\text {skim }} \simeq 3 \cdot 10^{12} \mathrm{molec} / \mathrm{s}$ \\
$T_{0}=296 \mathrm{~K}$ & $R e=5$ & $\mathcal{F}_{J=1, K=1} \simeq 1 \cdot 10^{11} \mathrm{molec} / \mathrm{s}$ \\
\hline
\end{tabular}

For the nuclear spin of the fluorine atom $(I=1 / 2), g_{I}$ reduces to

$$
g_{I}= \begin{cases}4 & \text { for } K \text { divisible by } 3 \text { (including zero) } \\ 2 & \text { for } K \text { not divisible by } 3\end{cases}
$$

Summarizing, by virtue of the BGC process, the occupation probability $p_{e_{g}, \boldsymbol{v}_{\boldsymbol{g}}, J=1, K=1} \simeq p_{J=1, K=1}$ of the $\left|e_{g}, \boldsymbol{v}_{g}, J=1, K=1\right\rangle$ level is enhanced by a factor of about 530 (compared to room temperature) up to $p_{J=1, K=1}(T=$ $4.2 \mathrm{~K})=3.5 \%$, thus yielding $\mathcal{F}_{e_{g}, \boldsymbol{v}_{g}, J=1, K=1} \triangleq \mathcal{F}_{J=1, K=1}=p_{J=1, K=1} \cdot \mathcal{F}_{\text {skim }} \simeq$ $1 \cdot 10^{11} \mathrm{molec} / \mathrm{s}$.

As argued, although some properties of a buffer gas beam can be varied by altering the flow and the hole size, certain applications require a finer control. In the following, we shall discuss two mutually exclusive manipulation methods which, according to the specific necessity, may be respectively implemented to reduce the temperature or the forward velocity of the beam.

\subsubsection{Further reduction of the beam temperature}

As already mentioned, in spite of a moderate rise in the mean longitudinal speed, a further temperature reduction can be achieved by increasing the gas pressure inside the cryogenic cell up to typical supersonic operating conditions. Characteristically, a supersonic expansion cools the internal degrees of freedom and reduces the width of the translational speed distribution with the price of a higher absolute speed in the laboratory frame. However, the resulting speed will be substantially lower in our case than for a conventional source, due to the lower temperature of the source $\left(T_{H e}\right)$. In other words, due 
to the more ordered velocity distribution established by the stagnation temperature inside the BGC cell, conversion into forward velocity of the beam will occur to a much lower extent. A quantitative treatment starts with the Bernoulli equation describing a stationary, adiabatic and irrotational flow of a compressible fluid in the absence of gravity, viscosity and shock waves [32]. Written in differential form for a fluid unit mass, this reads as

$$
u d u+\frac{d p}{\rho}=u d u+d h=0
$$

where $\rho$ and $h$ denote respectively the density and the enthalpy of the fluid, $u$ is the flow speed at a point on a streamline, and $p$ the pressure at the chosen point. Now, for a perfect gas we can write

$$
d h=c_{p} d T
$$

where $c_{p}=k_{B} \gamma / m(\gamma-1)$ represents the specific heat at a constant pressure, with $m$ and $\gamma$ being the gas mass and adiabatic index, respectively. Then, by inserting Eq. 13 into Eq. 12 and introducing the speed of sound

$$
a=\sqrt{\frac{\gamma k_{B} T}{m}}
$$

one derives the following expression for the sound velocity in an expanding gas

$$
a=\frac{a_{0}}{\sqrt{1+\frac{\gamma-1}{2} M^{2}}}
$$

where the Mach number $M=u / a$ has been introduced and $a_{0}$ is the initial sound velocity, i.e. inside the cell (note that $u_{0}=M_{0}=0$ ). We remark that, by definition, the Mach number is an increasing function of the distance from the orifice; indeed, while the beam velocity $u$ increases along the expansion path, the speed of sound $a$ diminishes due to the temperature decrease. Next, from Eq. 14, the following expansion law for $T$ is retrieved

$$
T=\frac{T_{0}}{1+\frac{\gamma-1}{2} M^{2}}
$$

while the velocity $u$ is given by

$$
u=M a=\frac{M a_{0}}{\sqrt{1+\frac{\gamma-1}{2} M^{2}}}
$$


Finally, the Poisson adiabatic, that is $p / p_{0}=\left(T / T_{0}\right)^{\gamma /(\gamma-1)}$ and $\rho / \rho_{0}=$ $\left(T / T_{0}\right)^{1 /(\gamma-1)}$, yields

$$
p=\frac{p_{0}}{\left(1+\frac{\gamma-1}{2} M^{2}\right)^{\frac{\gamma}{\gamma-1}}}
$$

and

$$
\rho=\frac{\rho_{0}}{\left(1+\frac{\gamma-1}{2} M^{2}\right)^{\frac{1}{\gamma-1}}}
$$

At this point, all the relevant parameters are known as a function of the Mach number. In turn, the dependence of the latter on the distance $x$ from a circular hole of radius $d$ can be expressed via the semi-empirical formula by Ashkenas and Sherman [33]

$$
M(x)=A\left(\frac{x-x_{0}}{2 d}\right)^{\gamma-1}-\frac{1}{2} \frac{(\gamma+1) /(\gamma-1)}{A\left[\left(x-x_{0}\right) /(2 d)\right]^{\gamma-1}}
$$

where $A$ and the ratio $x_{0} /(2 d)$ are known constants related to the gas species. As expected, the Mach number increases with $x$, but only up to the so-called freezing point where the density drops below a threshold value, which stops collisions and hence the cooling process. The maximum Mach number is found to be [34]

$$
M_{\max }=G\left(2 \sqrt{2} \sigma \rho_{0} d \epsilon\right)^{\frac{\gamma-1}{\gamma}}
$$

where $\epsilon$ represents the maximum fractional change in the mean velocity per collision, $\sigma$ the cross section for elastic collisions, and $G$ a proportionality constant also depending on the gas species. The minimum attainable temperature, $T_{\min } \equiv T\left(M_{\max }\right)$, is then calculated by inserting Eq. 21 into Eq. 16 ; the corresponding beam velocity, $u_{\max } \equiv u\left(M_{\max }\right)$, is obtained by plugging Eq. 21 into Eq. 17.

In the He case $\left(A \simeq 3.26, x_{0} \cdot(2 d)^{-1} \simeq 0.075, \gamma=5 / 3, G \simeq 2.03, \epsilon \simeq 0.02\right.$, and $\left.\sigma \simeq 3 \cdot 10^{-19} \mathrm{~m}^{2}\right)$ and for the above setup parameters, using a buffer gas flow of $100 \mathrm{SCCM}$ (corresponding to $\rho_{0} \simeq 3 \cdot 10^{23} \mathrm{~m}^{-3}$ ) leads to $T_{\min } \simeq 0.8$ $\mathrm{K}$ and $u_{\max } \simeq 210 \mathrm{~m} / \mathrm{s}$. Interestingly, while further increasing the product $\rho_{0} d$ still produces a corresponding decrease in $T_{\min }, u_{\max }$ tends to plateau $\left(u_{\text {max }}^{\text {plateau }}=227 \mathrm{~m} / \mathrm{s}\right)$; an excessive increment of $\rho_{0} d$ is however prevented by the onset of three-body collisions, which gives rise to clustering phenomena, 
as well as, via enhancement of the gas throughput, by an unmanageable background pressure, which gives rise to shock wave formation.

Finally, the extra enhancement in $p_{J=1, K=1}^{B G C, \text { sup }}$ (up to $21 \%$ ), brought about by the further temperature reduction (from 4.2 to $0.8 \mathrm{~K}$ ), would also provide a higher beam flux, $\mathcal{F}_{J=1, K=1}^{B G C, \text { sup }} \sim 6 \cdot 10^{11} \mathrm{molec} / \mathrm{s}$.

\subsubsection{Additional decrement of the beam speed}

Extra slowing of the beam can be accomplished by attaching a second cubicle to the aperture of the primary BGC cell. This second cubicle is also kept cold and equipped with gaps and vents through which the buffer gas can flow, creating a region of intermediate pressure (about $10 \%$ of that in the main cell) before the vacuum region [35]. In this way, the typical mean free path in the second cell is on the order of a few millimeters, so that $\mathrm{S}$ experiences only a few collisions. While preserving the low temperature, these few collisions will have the effect of lowering the boosted forward velocity of the molecules closer to the thermal speed. The price to pay is a tenable decrease in the molecular beam flow (between 1 and 2 orders of magnitude), against a reduction in the longitudinal speed by some factor. In this case, due to the lack of a reliable prediction model, the main beam parameters can only be characterized and optimized through comprehensive experimental investigations.

\subsection{Stark collimation of the $B G C$ beam}

On the basis of simple geometrical considerations, the beam emerging from the skimmer has a diameter of $2 r_{i}=2 \mathrm{~mm}$ and an angular divergence of $2^{\circ}$; thus, an electrostatic hexapole lens (EHL) will be used to minimize the spread in the transverse directions along the entire distance required for the Ramsey interrogation $(D=1 \mathrm{~m})$. For design purposes, we start by considering a beam of symmetric-top molecules which propagates along the $z$ axis of an $l_{\text {hex }}$-length hexapole. Then, to the first-order in the Stark interaction, the energy of a molecular rotational state with $K M_{E}<0$ increases with the electric field strength (here $M_{E}$ indicates the projection of $\boldsymbol{J}$ onto the local electric field vector); referred to as low-field-seeking (lfs), such a state experiences a force towards the electric field minimum region. In this case, the EHL matrix yielding the final radial displacement and velocity, $r\left(z=l_{\text {hex }}\right)$ and $u^{-1} \cdot v_{r}\left(z=l_{h e x}\right)$, in terms of the initial ones, $r_{i}=r(0)$ and $u^{-1} \cdot v_{r i}(z=0)$ (here $u$ denotes, as usual, the beam longitudinal speed), can be expressed as 
$[36]$

$$
\left(\begin{array}{cc}
\cos \left(p l_{\text {hex }}\right) & p^{-1} \sin \left(p l_{\text {hex }}\right) \\
-p \sin \left(p l_{\text {hex }}\right) & \cos \left(p l_{\text {hex }}\right)
\end{array}\right)
$$

where $p=(1 / u) \sqrt{\frac{\mu}{m} \frac{\left|K M_{E}\right|}{J(J+1)} \frac{6 V_{0}}{r_{0}^{3}}}, V_{0}$ being the applied potential and $r_{0}$ the hexapole radius. It is worth pointing out that the above matrix corresponds to the ideal hexapole where the six electrodes are extruded hyperbolas; in practice, this element is best approximated by parallel cylindrical (rod) electrodes having a radius that is 0.565 times that of the hexapole (see upper frame of Fig. 3) [37]. Next, we call $q$ the distance from the skimmer output to the hexapole entrance and $D$ the distance from the EHL output to the second Ramsey zone. Then, by applying the ABCD-matrix formalism, one can write

$$
\left(\begin{array}{c}
r(D) \\
u^{-1} \cdot v_{r}
\end{array}\right)=\left(\begin{array}{cc}
1 & D \\
0 & 1
\end{array}\right)\left(\begin{array}{cc}
\cos \left(p l_{\text {hex }}\right) & p^{-1} \sin \left(p l_{\text {hex }}\right) \\
-p \sin \left(p l_{\text {hex }}\right) & \cos \left(p l_{\text {hex }}\right)
\end{array}\right)\left(\begin{array}{ll}
1 & q \\
0 & 1
\end{array}\right)\left(\begin{array}{c}
r_{i} \\
u^{-1} \cdot v_{r i}
\end{array}\right)
$$

where the first and third matrices on the right-hand side account for the fact that molecules follow a straight-line trajectory in free space. Essentially, in order to collimate the molecular beam for a given $\left|J K M_{E}\right\rangle$ lfs state, one has to play with the $V_{0}, r_{0}, l_{\text {hex }}$ and $q$ parameters so as to set to zero the upperright element of the propagation matrix in Eq. 23. For the $\mid J=1, K=$ $\left.1, M_{E}=-1\right\rangle$ level, this happens in correspondence with the following choice: $q=5 \mathrm{~cm}, r_{0}=0.5 \mathrm{~cm}, V_{0}=5000 \mathrm{~V}, l_{\text {hex }}=1.6 \mathrm{~cm}$; then, $2 r(D=1 \mathrm{~m})=3.5$ $\mathrm{cm}$ and $v_{r}=1.4 \mathrm{~m} / \mathrm{s}$ are found. Actually, $l_{\text {hex }}$ does not represent the physical length of the hexapole lens (which is, in fact, greater), but rather the product $u t_{\text {hex }}$ where $t_{\text {hex }} \simeq 200 \mu \mathrm{s}$ is the time interval during which $V_{0}$ is applied; in this way, the hexapole field is switched on (off) when the molecules are far from the entrance (exit) so that the effects of fringe fields are negligible. For larger $V_{0}$ values, higher perturbative orders in the Stark interaction energy must be considered, whereupon the effect of an EHL is no longer simply described by Eq. 22. Moreover, for sufficiently intense electric fields, all rotational levels become high-field seeking (hfs); the corresponding states are deflected away from the $z$ axis and will eventually be lost. In order to identify the upper limit of the linear Stark-interaction regime so as to check the validity of Eq. 23, we calculated the exact expression (in the form of a rapidly convergent continued fraction) for the energy levels of a rigid symmetric top molecule in an external electric field of arbitrary strength 
[38]. As shown in Fig. 3, numerical evaluation carried out in the specific case of $\mathrm{CF}_{3} \mathrm{H}$ points out that the energy of the $\left|J=1, K=1, M_{E}=-1\right\rangle$ level ceases to change linearly only for field strengths exceeding $2.5 \mathrm{kV} / \mathrm{mm}$, while the lfs-to-hfs transition occurs above $6 \mathrm{kV} / \mathrm{mm}$.
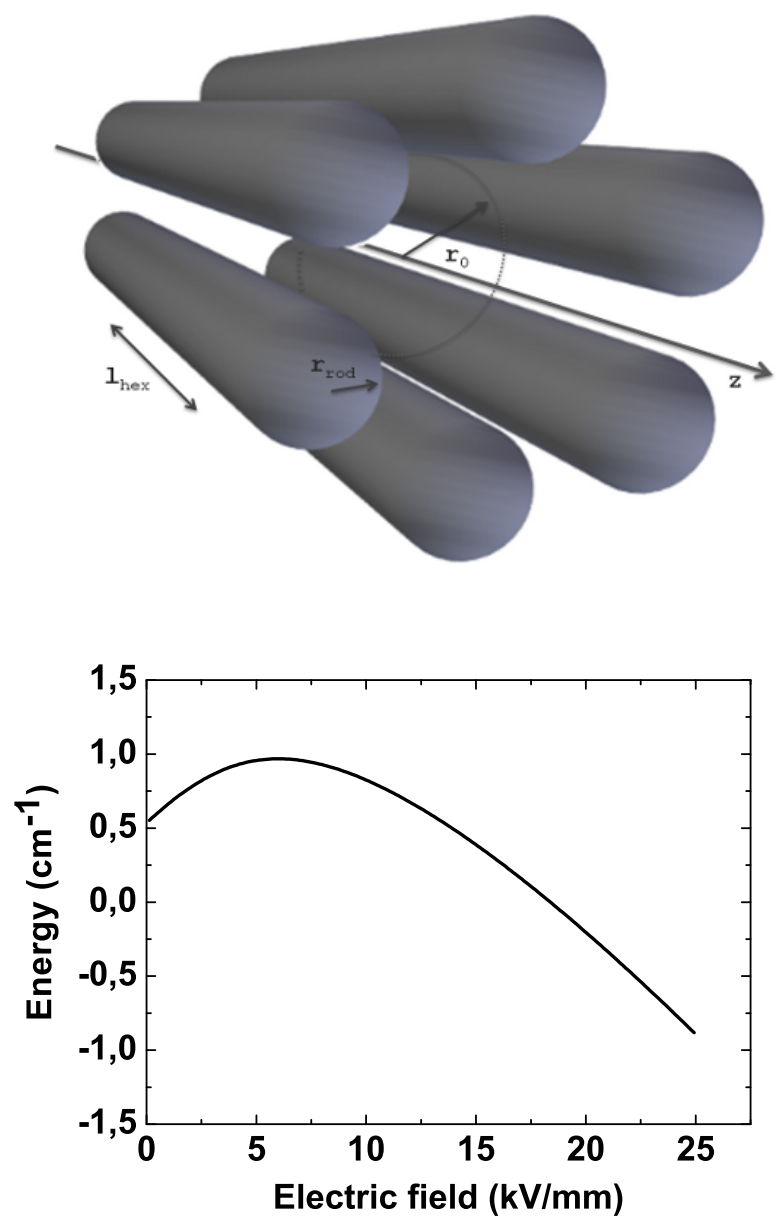

Figure 3: Upper frame: electrostatic-hexapole-lens realization by means of six rod electrodes, with radius $r_{\text {rod }}$, symmetrically arranged at distance $r_{0}$ from the beam $z$ axis. Lower frame: energy of the $\mathrm{CF}_{3} \mathrm{H}\left|J=1, K=1, M_{E}=-1\right\rangle$ level in an external electric field, evidencing the linear behavior below $2.5 \mathrm{kV} / \mathrm{mm}$ and the lfs-to-hfs transition around $6 \mathrm{kV} / \mathrm{mm}$. 


\section{Schematic layout of the whole experimental apparatus}

Summarizing, after the hexapole lens, the 4.2-K-temperature fluoroform beam should have the following characteristics:

- total flux in the lower rotational state of the selected two-photon transition: $\mathcal{F}_{J=1, K=1}^{\text {hex }}=(1 / 3)(1 / 3) \mathcal{F}_{J=1, K=1} \sim 1 \cdot 10^{10}$ molec $/ \mathrm{s}$. The first $1 / 3$ factor accounts for the fact that, among the three $|J=1, K=1\rangle$ Stark substates, only one, $\left|J=1, K=1, M_{E}=-1\right\rangle$, is low-field seeking; the second one is due to the pulsed operation of the EHL;

- mean forward velocity: $u \simeq 70 \mathrm{~m} / \mathrm{s}$;

- residual angular divergence: $\simeq 20 \mathrm{mrad}$.

As a result, the periodicity of the Ramsey fringes is $P=u /(2 D) \simeq 35 \mathrm{~Hz}$. In order to probe such a narrow spectroscopic feature with the necessary accuracy, an ultra stable and narrow laser source will be realized, referenced to an optical frequency comb (OFC). The whole experimental apparatus, under construction, is schematically shown in Fig. 4. Previously locked against the resonance of a high-finesse cavity made of ultra-low-expansion (ULE) glass, a continuous-wave (cw) 50-mW-power QCL (Thorlabs, QD8650CM) is referenced to an offset-free OFC covering a 4-THz-wide interval centered at 8.63 micron (with a power per tooth of $100 \mathrm{nW}$ ). The latter is produced via difference-frequency-generation (DFG) in a non-linear GaSe crystal [39] starting from the two outputs of a mode-locked Er-doped fiber laser oscillator (EDFO in Fig. 4), amplified in a custom dual-branch configuration (MenloSystems $\mathrm{GmbH}$ ). More in detail, the DFG pump laser beam comes from the main output which, consisting of an Er-doped fiber amplifier (EDFA) followed by a dispersion compensation system, provides $1.55-\mu \mathrm{m}$-wavelength pulses with a duration less than $70 \mathrm{fs}$ and an average output power of 340 $\mathrm{mW}$; the DFG signal beam comes from the second output that, comprising an independent EDFA plus a supercontinuum generation module, delivers an average output power greater than $300 \mathrm{~mW}$ over the entire spectrum $(1050-2100 \mathrm{~nm})$. In particular, a linewidth below $1 \mathrm{~Hz}$ is reached for the $8.63-\mu \mathrm{m}$ comb teeth by upstream stabilization (via a piezoelectric transducer and a faster intracavity electro-optic modulator) of the EDFO repetition rate (i.e. mode spacing) against a sub-Hertz-linewidth optical reference system [40]. The optical reference system is operated at Istituto Nazionale di Ricerca Metrologica (INRIM) in Turin and consists of a cw laser at $1.5 \mu \mathrm{m}$, locked 
to a high-finesse ULE glass cavity (clock laser) [41, 42]. In order to trace the final spectroscopic frequency measurement back to the Cs primary standard, the clock laser in Turin is linked to a Cs fountain (typical fractional frequency accuracy of $3 \cdot 10^{-16}$ and stability of $\left.2 \cdot 10^{-13} \cdot \tau^{-1 / 2}[44]\right)$. The clock laser is then delivered to our experiment in Florence via the national optical fiber link. In this way, phase locking to the 8.63-micron OFC [43] ensures that the QCL radiation has a stability better than $1 \mathrm{~Hz}$ up to $1000 \mathrm{~s}$, as required for data collection and averaging. Then, in order to enhance the signal-to-noise ratio in the final spectroscopic absorption signal, the QCL beam is split into two beams: one for the Ramsey excitation and the other for the detection; they are independently frequency shifted by two acoustooptic modulators in order to be resonant with the two-photon transition and the upper onephoton transition, respectively. Afterwards, the Ramsey beam is coupled to a U-shaped cavity (surrounded by a $\mu$-metal magnetic shield to avoid any fringe scrambling due to the Zeeman effect) having a finesse of several hundreds. As a result of the Ramsey excitation, the final, frequency-dependent probability for a molecule to be in the level $|\mathbf{2}\rangle$ is given by

$$
\mathcal{P}(\omega) \simeq \mathcal{B}(\omega)\left\{\mathcal{A}+\mathcal{C} \cos \frac{\omega-\omega_{20} / 2}{P}\right\}
$$

In the above equation, $\omega_{20} \equiv\left[E_{\text {ro-vib }}(|\mathbf{2}\rangle)-E_{\text {ro-vib }}(|\mathbf{0}\rangle)\right] / \hbar, \mathcal{B}(\omega)=D_{20}^{2}(\omega) \tau^{2}$, $\mathcal{A}=1+e^{-2 \gamma T}$, and $\mathcal{C}=2 e^{-\gamma T}$. Here, $T=D / u, \tau=d_{1-\text { zone }} / u$ is the interaction time in one single zone, and $\gamma$ represents the spontaneous lifetime of level $|\mathbf{2}\rangle$. Concerning the frequency-dependent amplitude, it can be expressed as $D_{20}(\omega)=\mu_{21} \mu_{10}|E|^{2} /\left[\hbar^{2}\left(\omega_{10}-\omega\right)\right] \equiv-\mu_{21} \mu_{10}|E|^{2} /(\hbar \delta)$, where $\omega_{10} \equiv\left[E_{\text {ro-vib }}(|\mathbf{1}\rangle)-E_{\text {ro-vib }}(|\mathbf{0}\rangle)\right] / \hbar,|E|^{2}$ is proportional to the intensity of the Ramsey laser beam, and $\mu_{21}\left(\mu_{10}\right)$ denotes the dipole matrix element corresponding to the transition $|\mathbf{2}\rangle \leftrightarrow|\mathbf{1}\rangle(|\mathbf{1}\rangle \leftrightarrow|\mathbf{0}\rangle)$. As anticipated, the transmission signal consists of periodical fringes, with period $P$, which are superimposed on the broader (Doppler-free) two-photon background signal $\mathcal{B}(\omega)$ arising from the absorption in one single zone. Then, further averaging of Eq. 24 over the distribution of molecular velocities is needed to obtain the actual signal; this will result in a gradual attenuation of the side fringes. The detection beam, instead, injects a separate Fabry-Perot cavity (with a finesse of a few thousand) to probe, via stimulated emission, the upper level population as induced by the Ramsey excitation and thus proportional to the actual fringe signal [45]. Eventually, a suitable function is fitted to the obtained fringe pattern to extract the periodicity and the center frequency. 
Regarding the latter, given the expected figures for the probe laser and the molecular beam, and for a reasonable signal-to-noise ratio $(S N R=25)$, averaging $N_{\text {avg }}=100$ individual measurements (each lasting several seconds) should return a resolution (accuracy) of $\sim P /\left(S N R \cdot \sqrt{N_{\text {avg }}}\right)=0.14 \mathrm{~Hz}$, corresponding to a fractional uncertainty of $4 \cdot 10^{-15}$. According to Eq. 3, this means that a comparison carried out over a few years would constrain the fractional temporal variation of the proton-to-electron mass ratio at the claimed level, provided that all the relevant systematic effects (second-order Doppler effect, ac Stark effect induced both by the blackbody radiation and the irradiated probe laser, collision shift, ... ) are properly taken into account and subtracted. This would represent an order-of-magnitude improvement compared to the current record, $\dot{\beta} / \beta=(-3.8 \pm 5.6) \cdot 10^{-14}[16]$.

\section{Conclusions}

We have defined the key stages for setting up an experiment which assesses the time constancy of the proton-to-electron mass ratio by precision ro-vibrational spectroscopy on a sample of cold molecules. In particular, crucial figures have been identified for both the molecular beam and the probe laser to constrain $\dot{\beta} / \beta$ at a level of some $10^{-15} /$ yr, resting on a two-photon Ramsey-fringes interrogation scheme. Starting with the molecular sample, the beam emerging from the BGC source will be soon characterized either by Resonance-Enhanced Multi-Photon Ionization (REMPI) or Cavity RingDown (CRD) spectroscopy. This will clarify the extent to which theoretical predictions are met and, hence, whether the hexapole lens is sufficient to reach all the molecular beam characteristics for an effective Ramsey interrogation or whether it is necessary to further implement one of the evolutionary strategies discussed in Sections 3.2.1 and 3.2.2. In this respect, it should be mentioned that another valuable option under consideration for additional slowing of the molecular beam, especially in view of future trapping applications, is represented by the so-called traveling-wave Stark decelerator [46]. In the meantime, after carrying out preliminary Fourier-Transform Infrared (FTIR) spectroscopy, high-resolution spectroscopic studies of fluoroform are planned in order to gain a deeper knowledge of the $8.63-\mu \mathrm{m}$ spectrum. On the other side, work is in progress for the realization of the OFC-assisted, QCL-based interrogation source. 


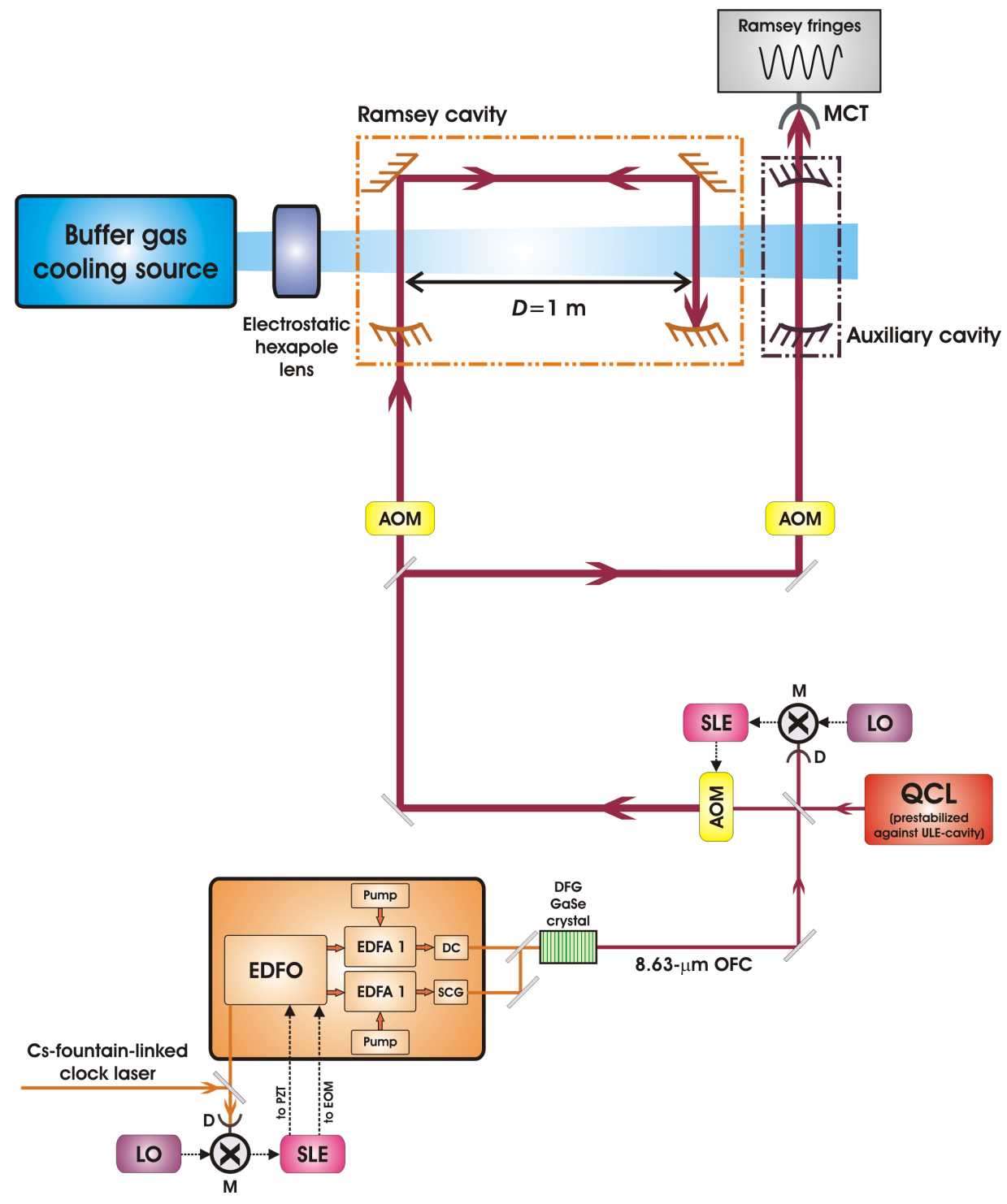

Figure 4: Schematic layout of whole experimental apparatus. The following legend holds: $\mathrm{DC}=$ dispersion compensation, $\mathrm{SCG}=$ supercontinuum generation, $\mathrm{AOM}=$ acoustooptic modulator, $\mathrm{LO}=$ local oscillator, $\mathrm{MCT}=\mathrm{HgCdTe}$ detector, $\mathrm{SLE}=$ servo loop electronics, $\mathrm{D}=\mathrm{InGaAs}$ detector, $\mathrm{M}=$ mixer.

\section{Acknowledgements}

The authors acknowledge helpful discussions with S.A. Meek and S. Skoff, and technical support by G. Notariale. This work was partly funded by 
MIUR-FIRB project RBFR1006TZ.

[1] C. Orzel, Phys. Scr. 86 (2012) 068101-9

[2] J.-P. Uzan, Rev. Mod. Phys. 75 (2003) 403-455

[3] S.G. Karshenboim, E. Peik, Lect. Notes Phys. 648 (2004) 1-18

[4] W.J. Marciano, Lect. Notes Phys. 648 (2004) 97-105

[5] C. Kiefer, Lect. Notes Phys. 648 (2004) 115-127

[6] S.N. Lea, Rep. Prog. Phys. 70 (2007) 1473-1523

[7] E. Peik, B. Lipphardt, H. Schnatz, Chr. Tamm, S. Weyers, R. Wynands, Proceedings of the 11th Marcel Grossmann Meeting on General Relativity, Eds.: H. Kleinert, R.T. Jantzen and R. Ruffini, World Scientific, Singapore (2008) 941-951

[8] V.V. Flambaum, A.F. Tedesco, Phys. Rev. C 73 (2006) 055501

[9] E. Reinhold, R. Buning, U. Hollenstein, A. Ivanchik, P. Petitjean, W. Ubachs, Phys. Rev. Lett. 96 (2006) 151101

[10] W. Ubachs, R. Buning, K.S.E. Eikema, E. Reinhold, Journal of Molecular Spectroscopy 241 (2007) 155-179

[11] P. Bonifacio, H. Rahmani, J.B. Whitmore, M.Wendt, M. Centurion, P. Molaro, R. Srianand, M.T. Murphy, P. Petitjean, I.I. Agafonova, S. D'Odorico, T.M. Evans, S.A. Levshakov, S. Lopez, C.J.A.P. Martins, D. Reimers, G. Vladilo, Astron. Nachr. 335 (2014) 1-9

[12] J. Bagdonaite, P. Jansen, C. Henkel, H.L. Bethlem, K.M. Menten, W. Ubachs, Science 339 (2013) 46-48

[13] H.L. Bethlem, M. Kajita, B. Sartakov, G. Meijer, W. Ubachs, Eur. Phys. J. Special Topics 163 (2008) 55-69

[14] D. Bakalov, V. Korobov, S. Schiller, Phys. Rev. A 82 (2010) 055401

[15] M. Kajita, New Journal of Physics 11 (2009) 055010 
[16] A. Shelkovnikov, R.J. Butcher, C. Chardonnet, A. Amy-Klein, Phys. Rev. Lett. 100 (2008) 150801

[17] L. Lorini, N. Ashby, A. Brusch, S. Diddams, R. Drullinger, E. Eason, T. Fortier, P. Hastings, T. Heavner, D. Hume, W. Itano, S. Jefferts, N. Newbury, T. Parker, T. Rosenband, J. Stalnaker, W. Swann, D. Wineland, J. Bergquist, Eur. Phys. J. Special Topics 163 (2008) 19-35

[18] N.F. Ramsey, Phys. Rev. 78 (1950) 695-699

[19] L.F. Constantin, R.J. Butcher, P.E. Durand, A. Amy-Klein, Ch. Chardonnet, Phys. Rev. A 60 (1999) R753-R756

[20] A. Amy-Klein, L.F. Constantin, R.J. Butcher, C. Daussy, P.E. Durand, G. Nogues, Ch. Chardonnet, Optics Express 4 (1999) 67-76

[21] S. Kondo, S. Saeki, J. Chem. Phys. 74 (1981) 6603-6611

[22] G. Herzberg, Molecular Spectra and Molecular Structure - II Infrared and Raman Spectra of Polyatomic Molecules (1991) Krieger Publishing Company, Malabar (Florida)

[23] R.W. Kirk, P.M. Wilt, Journal of Molecular Spectroscopy 58 (1975) $102-110$

[24] G. Klatt, A. Willetts, N.C. Handy, R. Tarroni, P. Palmieri, Journal of Molecular Spectroscopy 176 (1996) 64-74

[25] J.P. Champion, G. Graner, Mol. Phys. 58 (1986) 475-484

[26] S. Sofue, K. Kawaguchi, E. Hirota, T. Fujiyama, Bull. Chem. Soc. Jpn. 54 (1981) 3546-3550

[27] S.E. Maxwell, N. Brahms, R. deCarvalho, D.R. Glenn, J.S. Helton, S.V. Nguyen, D. Patterson, J. Petricka, D. DeMille, J. M. Doyle, Phys. Rev. Lett. 95 (2005) 173201

[28] N.R. Hutzler, H.-I Lu, J.M. Doyle, Chem. Rev. 112 (2012) 4803-4827

[29] L.D. van Buuren, C. Sommer, M. Motsch, S. Pohle, M. Schenk, J. Bayerl, P.W. H. Pinkse, G. Rempe, Phys. Rev. Lett. 102 (2009) 033001 
[30] D. Patterson, J.M. Doyle, J. Chem. Phys. 126 (2007) 154307

[31] N.E. Bulleid, S.M. Skoff, R.J. Hendricks, B.E. Sauer, E.A. Hinds, M.R. Tarbutt, Phys. Chem. Chem. Phys. 15 (2013) 12299

[32] G. Sanna, G. Tomassetti, Introduction to Molecular Beam Gas Dynamics (2005) Imperial College Press, London

[33] H. Ashkenas, F.S. Sherman, in IVth International Symposium on Rarefied Gas Dynamics, J. H. deLeeuw, ed. (Academic, New York, 1966), Vol. 2, p. 84

[34] M.D. Morse, Exp. Methods Phys. Sci. 29B (1996) 21-47

[35] H.-I Lu, J. Rasmussen, M.J. Wright, D. Patterson, J.M. Doyle Phys. Chem. Chem. Phys. 13 (2011) 18986-18990

[36] V.A. Cho, R.B. Bernstein, J. Phys. Chem. 95 (1991) 8129-8136

[37] S.Y.T. van de Meerakker, H.L. Bethlem, N. Vanhaecke, G. Meijer, Chem. Rev. 112 (2012) 4828-4878

[38] J.V. Hajnal, G.I. Opat, J. Phys. B: At. Mol. Phys. 24 (1991) 2799-2805

[39] A. Gambetta, R. Ramponi, M. Marangoni, Opt. Lett. 33 (2008) 26712673

[40] D.D. Hudson, K.W. Holman, R.J. Jones, S.T. Cundiff, J. Ye, D.J. Jones, Opt. Lett. 30 (2005) 2948-2950

[41] S.M. Foreman, K.W. Holman, D.D. Hudson, D.J. Jones, J. Ye Rev. Sci. Instrum. (2007) 78, 021101

[42] C. Clivati, A. Mura, D. Calonico, F. Levi, G.A. Costanzo, C.E. Calosso, A. Godone, IEEE Trans. Ultrason. Ferroelectr. Freq. Control (2011) $2582-2587$

[43] T. Sala, D. Gatti, A. Gambetta, N. Coluccelli, G. Galzerano, P. Laporta, M. Marangoni, Opt. Lett. 37 (2012) 2592-2594

[44] F. Levi, C. Calosso, D. Calonico, L. Lorini, E.K. Bertacco, A. Godone, G.A. Costanzo, B. Mongino, S.R. Jefferts, T.P. Heavner, E.A. Donley, IEEE Trans. Ultrason. Ferroelectr. Freq. Control (2010) 600-605 
[45] A. Shelkovnikov, C. Grain, R.J. Butcher, A. Amy-Klein, A. Goncharov, Ch. Chardonnet, IEEE JOURNAL OF QUANTUM ELECTRONICS 40 (2004) 1023-1029

[46] A. Osterwalder, S.A. Meek, G. Hammer, H. Haak, G. Meijer, Phys. Rev. A 81 (2010) 051401(R) 